\title{
Advancing the Science and Practice of Medication Adherence
}

\author{
Michael J. Stirratt, $P h D^{7}$, Jeffrey R. Curtis, MD, MS, MPH' ${ }^{2}$, Maria I. Danila, MD, MSc, MSPH', \\ Richard Hansen, PhD, RPh, FAPhA ${ }^{3}$, Michael J. Miller, RPh, DrPH, FAPhA ${ }^{4}$, and C. Ann Gakumo, PhD, RN ${ }^{5}$
}

'Division of AIDS Research, National Institute of Mental Health, Bethesda, MD, USA; ${ }^{2}$ Division of Clinical Immunology and Rheumatology, University of Alabama at Birmingham, Birmingham, AL, USA; ${ }^{3}$ Department of Health Outcomes Research and Policy, Auburn University Harrison School of Pharmacy, Auburn, AL, USA; ${ }^{4}$ Department of Pharmaceutical Sciences, Texas A\&M University Irma Lerma Rangel College of Pharmacy, College Station, TX, USA; ${ }^{5}$ Department of Acute, Chronic \& Continuing Care, University of Alabama at Birmingham School of Nursing, Birmingham, AL, USA.

\begin{abstract}
Medication adherence remains a significant unmet challenge for optimizing patient outcomes. Recent advances in the conceptualization, measurement, and support of medication adherence offer fresh opportunities to make a meaningful impact on adherence-related behavior and outcomes. These advances emphasize the multifaceted and dynamic nature of medication adherence, provide novel methods for monitoring medication adherence in clinical care, and articulate a set of multilevel strategies to more effectively improve and sustain medication adherence. Here, we offer recommendations for how clinicians can better engage with, and benefit from, these innovations to improve patient medication adherence and associated treatment outcomes.
\end{abstract}

KEYWORDS: adherence; persistence; adherence interventions; adherence measures; medication adherence.

J Gen Intern Med 33(2):216-22

DOI: $10.1007 /$ s11606-017-4198-4

(C) Society of General Internal Medicine 2017

\section{INTRODUCTION}

Improving adherence to medication has proven to be a vexing challenge. Large studies across multiple chronic conditions and drug classes have shown nonadherence rates of around $40-60 \%{ }^{1-3}$, suggesting that approximately one in every two prescription doses is not taken as prescribed. A recent Cochrane review on medication adherence interventions was not encouraging ${ }^{4}$ : among 182 randomized controlled trials of adherence interventions, the authors identified only five interventions that reported an impact on both adherence behavior and clinical outcomes, and these were characterized as providing only modest improvement and being complicated to implement. After the many years of research in the area of medication adherence, this is a disappointing conclusion.

What is needed to advance adherence research and practice? Productive future directions require an understanding of the current adherence landscape, which has been evolving in some important and positive ways. This narrative review will provide an overview of three current areas of innovation that may help advance the field - specifically, advances in the conceptualization of adherence, new tools for adherence measurement and monitoring, and novel intervention approaches.

Published online December 4, 2017

\section{ADVANCING THE CONCEPTUALIZATION OF MEDICATION ADHERENCE}

Medication adherence has been defined as "the extent to which patients take medications as prescribed by their health care providers" ${ }^{5}$. This construct can be operationalized in many ways. Better and more consistent conceptualization of medication adherence provides an important foundation for advancing adherence, and it informs our future directions for both research and clinical practice.

First, it is important to distinguish medication adherence behavior within the context of several related constructs, such as patient knowledge or understanding of a medication regimen, reasons for nonadherence, or attitudes and beliefs about medications. These constructs are better recognized as important antecedents or consequences of medication adherence behavior ${ }^{6},{ }^{7}$.

Second, medication adherence needs to be understood as a multidimensional construct. Much adherence research has focused on patient implementation of medication regimens in terms of doses taken or days covered. There is more to adherence — particularly when we think about adherence as a process enacted over time. A scientific consensus group has described three core components of medication adherence: initiation (i.e., starting a recommended medication regimen), implementation (i.e., executing the prescribed dosage schedule), and persistence (i.e., length of time on regimen before discontinuation) ${ }^{8}$.

Initiation refers to the point when a patient begins taking a medication. Primary nonadherence occurs when a patient has been prescribed a medication but never fills it ${ }^{9}$. Primary nonadherence has also been described as initial medication adherence; both terms seek to differentiate it from aspects of medication adherence that occur following drug initiation ${ }^{10}$. Although this adherence domain is comparatively understudied, evidence across a variety of drug classes and chronic illnesses suggests that approximately one-quarter of patients never fill and initiate a new prescription ${ }^{9}$.

Implementation refers to actual dose-taking, and this behavior is most often associated with the term adherence. Regimen implementation may be operationalized in terms of the proportion of pills that were taken relative to the number of pills prescribed. A meta-analysis of 569 studies of health-related 
behaviors, including medication-taking, revealed that patients took $75.2 \%$ of doses on average ${ }^{11}$. Regimen implementation is also commonly measured through prescription refill-based approaches such as the medication possession ratio (MPR) and proportion of days covered (PDC) ${ }^{12-14}$.

Persistence refers to adherence over time, and whether patients are taking a medication continuously over longer intervals. Roughly $40 \%$ of patients across various chronic diseases and conditions discontinue medication within 1 year ${ }^{15}$. Measuring persistence with an overall treatment regimen, rather than a specific medication, may be needed. In a large cohort of individuals with acute myocardial infarction (MI), 31\% discontinued the use of secondary prevention therapies such as aspirin and betablockers within 6 months ${ }^{16}$.

The multiple aspects of adherence can be somewhat independent. For example, a study conducted among patients with rheumatoid arthritis treated with tumor necrosis factor (TNF) inhibitors found that nearly $80 \%$ remained persistent with therapy over 3 years, but regimen implementation was generally low, with only 25-37\% of patients achieving an MPR of $0.80{ }^{17}$, meaning that patients failed to take their medication appropriately on a daily basis, but continued using their medication in general.

Finally, in addition to recognizing multiple dimensions of medication adherence, adherence must be understood as dynamic rather than static. Clinicians and researchers tend to view adherence as a stable and dichotomous property of individuals, i.e., "there are adherent patients, and there are non-adherent patients." This misses the fact that medication adherence in chronic conditions may vary in response to disease activity, treatment methods, and the course of psychosocial comorbidities. Longitudinal data has identified a tendency for regimen implementation to decline over time ${ }^{18},{ }^{19}$, as well as distinct longitudinal patterns of medication use among patient subgroups (i.e., dose-taking that is consistently high, consistently low, increasing, decreasing, or variable) ${ }^{20-}$ 22 . A large HIV patient cohort showed high regimen implementation over extended intervals, but $48 \%$ of patients had at least one 6-month period of low adherence over a median 4.5 years of follow-up ${ }^{23}$. Longitudinal changes in adherence among HIV patients have been associated with concurrent changes in life stress, substance use, and medication beliefs ${ }^{24}$. Medication use can also be episodic: most osteoporosis patients discontinue pharmacotherapy at some point but reinitiate it after an extended gap ${ }^{25}$. Short gaps in antidepressant medication supply are common and are predictive of discontinuance among individuals with major depressive disorders ${ }^{26}$.

Therefore, recognizing that medication adherence is multidimensional and dynamic, what are the implications for clinical practice and research? First, each dimension of adherence (initiation, implementation, and persistence) may have unique determinants, and may require distinct interventions. Second, adherence dynamics underscore the need for routine monitoring of medication adherence in clinical practice. To make effective clinical decisions, providers need data to determine whether patients have initiated pharmacotherapy and how well they are implementing and persisting with the regimen. Timely and accurate knowledge of medication adherence are critical for informing clinical decisions about the need for dose escalation, regimen changes, or interventions to address adherence behavior. Third, the dynamic adherence concept also supports the notion of "critical junctures"-periods when monitoring and supporting adherence are particularly important (e.g., early in the course of therapy, after experiencing treatment failure, following hospital discharge).

\section{ADVANCING THE MEASUREMENT OF MEDICATION ADHERENCE}

Advances in measurement frequently precede advances in science and medicine. This applies to adherence research and practice as well. New innovations in adherence measurement are currently under way and will provide fresh capacity for real-time monitoring and more precise estimation. The ultimate goal is to transform medication adherence into a "vital sign" to which clinicians routinely attend ${ }^{27}$.

The major adherence measurement methods have been cataloged and described previously ${ }^{28-30}$. These chiefly encompass patient self-reports of adherence, prescription refill measures, pill counts, and electronic drug monitors. No single adherence measurement approach is perfect. All have advantages and disadvantages, depending on their context and purpose of use ${ }^{31},{ }^{32}$.

The proliferation of electronic devices for measuring medication adherence is evident in the many types of pill bottles, pill organizers, and blister packs with an electronic interface 33. These devices measure when a medication was accessed to generate date- and time-stamped implementation data. Electronic drug monitors with wireless capability can transmit their data in real time to facilitate timely interventions in response to observed nonadherence. While the use of these devices is increasingly straightforward for patients, the ready integration of electronic device data into primary care has a long way to go; some of these approaches might be more feasibly implemented in pharmacy settings. These electronic devices also cannot ensure that a pill was ingested, only that it was removed from the bottle, pill organizer, or blister pack.

Ingestible sensors are a newer method of measuring adherence directly. They represent a significant innovation by providing definitive evidence of pill ingestion in real time. A typical biosensor has a radio frequency ID-tagged sensor embedded within a gelatin capsule, which can be coencapsulated with medication by a pharmacist. When the capsule reaches the stomach, its coating dissolves, and the biosensors relay information to a sensing device worn by the patient. The data is then wirelessly transferred to a HIPAAcompliant cloud-based server. An ingestible sensor system developed by Proteus Digital Health (Redwood City, CA) ${ }^{34}$ has been approved by the Food and Drug Administration (FDA). A specific proposal for co-formulation of an 
antipsychotic medication embedded within the Proteus ingestible sensor is under regulatory review. Studies to date support ingestible sensor safety ${ }^{34}$, acceptability ${ }^{35},{ }^{36}$, and improved measurement precision over self-report ${ }^{37}$. How widely these new technologies will be used remains an open question.

Rapid point-of-care drug assays that can characterize medication adherence would be a closer fit to current care practices. Biochemical assessment of antihypertensive drug adherence through mass spectrometry with urine and serum samples has demonstrated a clear relationship between nonadherence and elevated blood pressure ${ }^{38},{ }^{39}$. Dried blood spots (DBS) have been used to quantify long-half-life antiretroviral drug metabolites in a manner that can characterize medication adherence over several weeks ${ }^{40}$. This overcomes the problem of "white-coat adherence" (only taking medicines prior to a clinical or research appointment) that can plague plasma drug concentration measurement. Further research to advance such assays, and to make them rapid and deliverable at the point of care, is under way in many areas.

Until new adherence technologies and assays can be better integrated into clinical care, patient self-reports will remain a primary approach for obtaining information about medication adherence. Self-reports are known to be imprecise and to overestimate adherence, but progress has been made in developing questions and systems for more accurately collecting self-reported adherence data ${ }^{41}$. Systematic computer collection of patient-reported outcomes (PROs) can improve the validity of self-reported adherence data and make it readily available to providers when integrated into the electronic health record (EHR). In one example from a network of HIV clinics, patients in the waiting room received a computer tablet to complete a questionnaire regarding medication adherence and other PROs ${ }^{42},{ }^{43}$. Answers to the adherence question predicted biological treatment outcomes (viral load and CD4 counts) ${ }^{42}$; they also were demonstrably helpful for clinicians in identifying patients in need of adherence support ${ }^{43}$. Routine computer collection of adherence PROs through brief, validated measures is a standard to which we should aspire.

In the absence of other systematic approaches to adherence monitoring, providers can improve an old standby: patientprovider communication. There are better ways and worse ways to ask patients about adherence. Directive and leading questions are unhelpful (e.g., "You are taking your medications, right?") ${ }^{44}$. The provider should instead ask open-ended, non-judgmental questions to start a conversation about adherence (e.g., "How do you think these medicines are working for you? What has it been like to take these medications? What's the hardest thing about taking these medications?"), and follow up with at least one clarifying question so that the patient feels heard ${ }^{45}$. If concerns about possible nonadherence remain, the clinician should then ask directly ("When was the last time you missed a dose?"), because negatively framed follow-up questions appear to engender more accurate responses ${ }^{44}$. The alternative to asking these types of questions during a busy clinical encounter would be the use of a validated questionnaire that the patient could complete individually. Many examples are available, including questionnaires that specifically ask about barriers to adherence ${ }^{41}$.

Improved "diagnosis" of medication nonadherence represents a fundamental action point for delivery of interventions. We have touched upon a number of recent advances in medication adherence monitoring and measurement: wireless monitors, ingestible sensors, drug assays, computer-based PROs, and patient self-reports during clinical encounters. These modalities all advance the crucial goal of integrating more routine and improved monitoring of adherence into clinical practice. The medication monitoring approaches vary substantially in cost and ease of implementation in clinical settings. It may be necessary to pursue comparatively low-cost practices that routinely survey patients on adherence (e.g., careful patient-provider communication, computer collection of PROs) and to reserve more specialized and intensive methods (e.g., wireless monitors, ingestible sensors) for patients at critical junctures who are in need of closer adherence monitoring and support. In the future, clinicians might prescribe electronic adherence monitoring for selected patients, much like they currently prescribe a medication or order laboratory monitoring ${ }^{46}$. Clinicians can also make referrals for provision of adherence support through allied providers or other available services.

\section{ADVANCING INTERVENTIONS TO IMPROVE ADHERENCE}

With improved conceptualization and monitoring of medication adherence, we are better positioned to advance effective interventions that improve and sustain adherence. In light of the many limitations of the available adherence intervention literature ${ }^{4}$, we would like to stress that adherence interventions can be simple and effective. "Proof of concept" for this idea can be seen in two adherence intervention trials, both of which demonstrated an impact on adherence and hard outcomes.

One of the first trials to demonstrate that mobile health (mHealth) approaches could improve adherence was published by Lester et al. ${ }^{47}$. The "WelTel" trial tested a simple, patient-centered, interactive text messaging approach to engage individuals with HIV as they started antiretroviral treatment in Kenya. Investigators sent study participants a once-aweek text message that simply asked, "How are you?" Participants were asked to respond to this message in one of two ways: "fine" or "problem." Those who said "problem" would get a call from a nurse, who would address their medicationrelated concerns. Participants who engaged in the text messaging protocol for 1 year not only had better self-reported adherence, but also had higher rates of viral suppression, the biological correlate of HIV medication adherence ${ }^{47}$. 
A study by Choudhry et al. demonstrated how an intervention in care systems can affect adherence ${ }^{48}$. The trial included 5855 individuals who had experienced a major vascular event and who were followed to examine adherence under two conditions: no co-pay (full coverage for treatment) or standard co-pay. Although those who received full coverage had only modestly better refill adherence, there was a particularly important impact on outcomes related to racial disparities, including a $35 \%$ reduction in vascular events in patients of color and a $70 \%$ reduction in health care spending 48 .

So how should we move forward with adherence interventions? These examples show that such interventions can have a meaningful effect — yet we will not likely find a "silver bullet" or a "one-size-fits-all" solution. Since any single approach may be modestly helpful, and the best intervention may differ by patient, effective adherence support will require a combination of interventions. An ecological approach that advances adherence interventions at multiple levels, including the drug regimen, the patient, the provider, and the care system, will be necessary. This multilevel approach takes into consideration the interdependence between a patient and their surrounding environment.

\section{Regimen Level}

For patients with increasingly complex medication regimens, simplification of these regimens can help improve adherence. Efforts to combine multiple drugs into a single "polypill" with once-daily dosing may reduce the burden of adherence 49 . Long-acting regimens, such as extended-release or sustainedrelease preparations ${ }^{50}$, may also be helpful by reducing the dosing frequency and improving medication tolerability. Pharmacy-based efforts to synchronize medication refill dates provide further simplification for the patient ${ }^{51}$, and have been associated with improved regimen implementation in terms of the MPR ${ }^{52}$.

\section{Patient Level}

There has long been the notion that nonadherence is chiefly about forgetfulness on the part of the patient, and that reminders to take medications will thus be helpful. Unfortunately, reminders alone can come up short. A major randomized trial that enrolled more than 50,000 participants and tested multiple types of basic medication reminder devices (e.g., pillboxes, pill bottle caps with timers) recently reported finding no impact on medication adherence ${ }^{53}$. However, questions were raised as to how broadly and persistently the devices were used, in addition to their capacity for impact. We should not reject the use of pillbox organizers and other devices on this basis, but should recognize that their use alone may not be sufficient.

There is evidence that patient-directed counseling approaches can improve and sustain medication adherence. A review of adherence intervention trials found that case management, medication therapy management, and programs that provided patient education and behavioral support were among the few approaches with documented improvement in patient implementation of medication regimens in multiple areas of chronic illness ${ }^{54}$. This research finding reflects a continuing need to work closely with patients on their individual psychosocial concerns and challenges in order to have an impact on adherence ${ }^{55}$. Patient-directed counseling approaches also suggest a team approach, as social workers, behavioral health providers, clinical pharmacists, and adherence specialists have important roles to play in the delivery of these intervention approaches.

Substantial research on patient-directed $\mathrm{mHealth}$ adherence interventions is advancing, and carefully designed, interactive, patient-centered approaches show a real effect. A variety of interventions delivered through mHealth technologies such as text messaging or smartphone applications have shown improvements in medication adherence ${ }^{56}$. In addition to the previously cited WelTel trial among HIV patients ${ }^{47}$, mHealth interventions have reported an effect on hard outcomes in areas such as hypertension ${ }^{57}$. The Smartphone Medication Adherence Stops Hypertension (SMASH) program, which delivered different motivational text messages contingent upon levels of electronically monitored regimen implementation, showed a significant favorable effect on blood pressure ${ }^{57}$. It is interesting to note that both WelTel and SMASH transcended simple text message reminders and instead delivered highly interactive, ongoing, patient-centered care. This inclusion of patient-centered care speaks to the unique capacities and strengths of mHealth tools. While these mHealth interventions may be unlikely to be adopted by individual providers, they can be a valuable tool for clinics, comprehensive care teams, and collaborating pharmacies in supporting adherence.

\section{Provider Level}

We have underscored how improved adherence monitoring can enable providers to respond in appropriate ways. Providing clinicians with a brief adherence report based on electronic drug monitors was shown to significantly improve appropriate clinical management for patients with uncontrolled hypertension-specifically, regimen intensification for adherent patients and adherence counseling for non-adherent patients 58. We have also seen improved provider response when receiving adherence data in the EHR via computer-collected PROs $^{43}$. In the hypertension trial, adherence reports improved ratings of clinician communication and patient-centered care 58; however, this is a point that requires caution. One trial that provided clinicians with brief adherence reports based on electronic drug monitoring increased unhelpful communication ("finger-wagging") rather than increasing patient implementation of the drug regimen ${ }^{59}$. It is important for providers to respond in a constructive and nonjudgmental manner, as detailed earlier. Improving provider communication skills and attention to shared decision-making with patients can increase adherence $^{60}, 61$. 


\section{System Level}

System-level interventions involve aspects of health care delivery and coverage, and represent an opportunity to address adherence at the broad, population level. There is growing evidence of the importance of these health care system factors for adherence and the capacity to modify them with beneficial impact. Reduced co-pays have been found to increase medication adherence across a variety of chronic conditions ${ }^{54}$, perhaps by reducing barriers to prescription fills and persistent refill. Other helpful care system approaches include pharmacy-based tools such as medication refill date synchronization, extended prescription length, and use of mail-order pharmacies ${ }^{62}$. Systems approaches can further involve the composition of care teams. An analysis of comprehensive care teams within the Kaiser Permanente system found higher medication adherence among HIV patients when the care teams included clinical pharmacists and social workers ${ }^{63}$.

Multilevel approaches addressing the regimen, patient, provider, and health care system can combine and magnify the impact of any single approach. Clinicians alone cannot advance all of these approaches, of course. What is chiefly under the control of the clinician is directing the regimen, assessing adherence, and responding accordingly. Providers who engage in constructive and nonjudgmental communication with patients can explore barriers to adherence ("What do you think gets in the way of taking your medicines?"). The impulse to prescribe solutions is strong but should be resisted; it is better to elicit answers from the patient ("What are some possible solutions to that challenge?"). A team approach is also important; clinicians should help connect patients to behavioral health care and support services when indicated.

\section{CONCLUSION: FUTURE DIRECTIONS}

Advances in our understanding and monitoring of adherence, and in developing interventions to support adherence, are crucial to making a greater impact in this domain. We have outlined advancements in each area. These areas underscore how adherence is multifaceted and dynamic, requires regular monitoring, and will benefit from multilevel approaches.

Future efforts should address improved integration of adherence monitoring into clinical practice. Technology-based measures including patient-reported surveys and wireless monitoring systems must be developed such that they can be appropriately integrated within the EHR and provide clinicians with clear and timely information on patient adherence. Research to better identify critical junctures for intensified adherence monitoring would be well warranted. With further longitudinal research and adherence monitoring in patient care, the development of predictive analytics to identify patients at risk for poor adherence or non-persistence could advance the delivery of proactive interventions to prevent these outcomes.
There is a continuing need to deepen and strengthen the evidence base for adherence support interventions. Adherence interventions should consider the specific form of adherence (initiation, implementation, persistence) that they seek to target, since each may have its own unique determinants, facilitators, and barriers. A critical element in developing adherence interventions is achieving a practical design that can be easily incorporated into routine medical care and practice, and in individuals' day-to-day lives outside the clinical setting. These interventions may need to recognize the many time demands placed on primary care providers, and may rely on a multidisciplinary team (nurses, clinical pharmacists, social workers) for implementation. Individual-level counseling interventions will be needed, but they can be difficult to deliver at scale unless they are properly reimbursed. If the cost of medication nonadherence is high, some consideration should be given to reimbursement for services, such as medication therapy management, that will reduce the nonadherence cost burden. mHealth interventions can be powerful tools, given their capacity to deliver interactive, tailored, real-time intervention. However, further improvement in medication adherence cannot be achieved by technological advancements alone. We will need to work on multiple levels, and recognize that the best approach for each patient will be unique and tailored to their individual needs.

Acknowledgements: Dr. Gakumo is supported by the Robert Wood Johnson Foundation Nurse Faculty Scholars Program 72113. Dr. Danila is supported by NIAMS K23 AR062100. The authors would like to thank the UAB "Interdisciplinary Approaches to Understanding and Improving Adherence" conference leaders, and Elizabeth Rahn, $\mathrm{PhD}$, for their review and input in the preparation of this manuscript, and Stacey C. Tobin, PhD, for providing editorial support. Appreciation is also extended to members of the NIH Adherence Network for input on a related $U A B$ conference presentation.

Corresponding Author: Michael J. Stirratt, PhD; Division of AIDS Research National Institute of Mental Health, Bethesda, MD, USA (e-mail: stirrattm@mail.nih.gov).

\section{Compliance with Ethical Standards:}

Conflict of Interest: Drs. Stirratt, Gakumo, Curtis, Danila, and Miller report no conflict of interest. In the past 3 years, Dr. Hansen has provided expert testimony for Boehringer Ingelheim on unrelated matters.

\section{REFERENCES}

1. Briesacher BA, Andrade SE, Fouayzi H, Chan KA. Comparison of drug adherence rates among patients with seven different medical conditions. Pharmacotherapy. 2008;28(4):437-43.

2. Vink NM, Klungel $\mathbf{O H}$, Stolk RP, Denig P. Comparison of various measures for assessing medication refill adherence using prescription data. Pharmacoepidemiol Drug Saf. 2009; 18(2): 159-65.

3. Yeaw J, Benner JS, Walt JG, Sian S, Smith DB. Comparing adherence and persistence across 6 chronic medication classes. J Manag Care Pharm. 2009; 15(9):728-40.

4. Nieuwlaat $\mathbf{R}$, Wilczynski $\mathbf{N}$, Navarro $\mathbf{T}$, et al. Interventions for enhancing medication adherence. Cochrane Database Syst Rev. 2014(11):CD000011.

5. Osterberg L, Blaschke T. Adherence to medication. N Engl J Med. 2005;353(5):487-97. 
6. Steiner JF. Self-reported adherence measures: what do they assess and how should we use them? Med Care. 2012;50(12):1011-2.

7. Voils CI, Hoyle RH, Thorpe CT, Maciejewski ML, Yancy WS, Jr. Improving the measurement of self-reported medication nonadherence. J Clin Epidemiol. 2011;64(3):250-4

8. Vrijens B, De Geest S, Hughes DA, et al. A new taxonomy for describing and defining adherence to medications. $\mathrm{Br} \mathrm{J}$ Clin Pharmacol. 2012;73(5):691-705.

9. Fischer MA, Stedman MR, Lii J, et al. Primary medication nonadherence: analysis of 195,930 electronic prescriptions. J Gen Intern Med. 2010;25(4):284-90.

10. Hutchins DS, Zeber JE, Roberts CS, et al. Initial medication adherencereview and recommendations for good practices in outcomes research: an ISPOR medication adherence and persistence special interest group report. Value Health. 2015;18(5):690-9.

11. DiMatteo MR. Variations in patients' adherence to medical recommendations: a quantitative review of 50 years of research. Med Care. 2004;42(3):200-9.

12. Curtis JR, Xi J, Westfall AO, et al. Improving the prediction of medication compliance: the example of bisphosphonates for osteoporosis. Med Care. 2009;47(3):334-41.

13. Hess LM, Raebel MA, Conner DA, Malone DC. Measurement of adherence in pharmacy administrative databases: a proposal for stan dard definitions and preferred measures. Ann Pharmacother. 2006;40(78):1280-88

14. Steiner JF, Prochazka AV. The assessment of refill compliance using pharmacy records: methods, validity, and applications. J Clin Epidemiol. 1997;50(1):105-16.

15. Blaschke TF, Osterberg L, Vrijens B, Urquhart J. Adherence to medications: insights arising from studies on the unreliable link between prescribed and actual drug dosing histories. Annu Rev Pharmacol Toxicol. 2012;52:275-301

16. Mathews R, Wang TY, Honeycutt E, et al. Persistence with secondary prevention medications after acute myocardial infarction: Insights from the TRANSLATE-ACS study. Am Heart J. 2015;170(1):62-9.

17. Calip GS, Adimadhyam S, Xing S, Rincon JC, Lee WJ, Anguiano RH Medication adherence and persistence over time with self-administered TNF-alpha inhibitors among young adult, middle-aged, and older patients with rheumatologic conditions. Semin Arthritis Rheum. 2017. https://doi.org/10.1016/j.semarthrit.2017.03.010

18. Benner JS, Glynn RJ, Mogun H, Neumann PJ, Weinstein MC, Avorn J. Long-term persistence in use of statin therapy in elderly patients. JAMA. 2002;288(4):455-61.

19. Wilson IB, Bangsberg DR, Shen J, et al. Heterogeneity among studies in rates of decline of antiretroviral therapy adherence over time: results from the multisite adherence collaboration on HIV 14 study. J Acquir Immune Defic Syndr. 2013;64(5):448-54

20. Hommel KA, McGrady ME, Peugh J, et al. Longitudinal patterns of medication nonadherence and associated health care costs. Inflamm Bowel Dis. 2017;23(9): 1577-83.

21. Knafl GJ, Bova CA, Fennie KP, O'Malley JP, Dieckhaus KD, Williams AB. An analysis of electronically monitored adherence to antiretroviral medications. AIDS Behav. 2010;14(4):755-68.

22. Franklin JM, Shrank WH, Pakes J, et al. Group-based trajectory models: a new approach to classifying and predicting long-term medication adherence. Med Care. 2013;51(9):789-96.

23. Cambiano V, Lampe FC, Rodger AJ, et al. Long-term trends in adherence to antiretroviral therapy from start of HAART. AIDS (London, England). 2010;24(8):1153-62.

24. French T, Tesoriero J, Agins B. Changes in stress, substance use and medication beliefs are associated with changes in adherence to HIV antiretroviral therapy. AIDS Behav. 2011;15(7):1416-28.

25. Cadarette SM, Burden AM. Measuring and improving adherence to osteoporosis pharmacotherapy. Curr Opin Rheumatol. 2010;22(4):397 403.

26. Hansen RA, Dusetzina SB, Dominik RC, Gaynes BN. Prescription refill records as a screening tool to identify antidepressant non-adherence. Pharmacoepidemiol Drug Saf. 2010;19(1):33-7.

27. Vrijens B, Antoniou S, Burnier M, de la Sierra A, Volpe M. Current situation of medication adherence in hypertension. Front Pharmacol 2017;8:100

28. Berg KM, Arnsten JH. Practical and conceptual challenges in measuring antiretroviral adherence. J Acquir Immune Defic Syndr. 2006;43 Suppl $1:$ S79-87.

29. Gonzalez JS, Schneider HE. Methodological issues in the assessment of diabetes treatment adherence. Curr Diab Rep. 2011;11(6):472-9.
30. Whalley Buono E, Vrijens B, Bosworth HB, Liu LZ, Zullig LL, Granger BB. Coming full circle in the measurement of medication adherence: opportunities and implications for health care. Patient Prefer Adherence. 2017;11:1009-17.

31. Chesney MA. The elusive gold standard. Future perspectives for HIV adherence assessment and intervention. J Acquir Immune Defic Syndr. 2006;43 Suppl 1:S149-55

32. Zullig LL, Mendys $\mathbf{P}$, Bosworth HB. Medication adherence: A practical measurement selection guide using case studies. Patient Educ Couns. 2017;100(7):1410-4

33. Campbell JI, Haberer JE. Cell Phone-Based and Adherence Device Technologies for HIV Care and Treatment in Resource-Limited Settings: Recent Advances. Curr HIV/AIDS Rep. 2015;12(4):523-31.

34. Hafezi H, Robertson TL, Moon GD, Au-Yeung KY, Zdeblick MJ, Savage GM. An ingestible sensor for measuring medication adherence. IEEE Trans Biomed Eng. 2015;62(1):99-109

35. Kane JM, Perlis RH, DiCarlo LA, Au-Yeung K, Duong J, Petrides G. First experience with a wireless system incorporating physiologic assessments and direct confirmation of digital tablet ingestions in ambulatory patients with schizophrenia or bipolar disorder. J Clin Psychiatry. 2013;74(6):e533-40.

36. Peters-Strickland T, Pestreich L, Hatch A, et al. Usability of a novel digital medicine system in adults with schizophrenia treated with sensorembedded tablets of aripiprazole. Neuropsychiatr Dis Treat. 2016;12:2587-94

37. Thompson D, Mackay T, Matthews M, Edwards J, Peters N, Connolly SB. Direct adherence measurement using an ingestible sensor compared with self-reporting in high-risk cardiovascular disease patients who knew they were being measured: a prospective intervention. JMIR Mhealth Uhealth. 2017;5(6):e76.

38. Gupta P, Patel P, Strauch B, et al. Risk Factors for Nonadherence to Antihypertensive Treatment. Hypertension. 2017;69(6):1113-20.

39. McNaughton CD, Brown NJ, Rothman RL, et al. Systolic Blood Pressure and Biochemical Assessment of Adherence: A cross-sectional analysis in the emergency department. Hypertension. 2017;70(2):307-14.

40. Zheng JH, Rower C, McAllister K, Castillo-Mancilla J, et al. Application of an intracellular assay for determination of tenofovir-diphosphate and emtricitabine-triphosphate from erythrocytes using dried blood spots. J Pharm Biomed Anal. 2016;122:16-20.

41. Stirratt MJ, Dunbar-Jacob J, Crane HM, et al. Self-report measures of medication adherence behavior: recommendations on optimal use. Trans Behav Med. 2015;5(4):470-82

42. Feldman BJ, Fredericksen RJ, Crane PK, et al. Evaluation of the single-item self-rating adherence scale for use in routine clinical care of people living with HIV. AIDS Behav. 2013;17(1):307-18

43. Crane HM, Crane PK, Tufano JT, et al. HIV provider documentation and actions following patient reports of at-risk behaviors and conditions when identified by a web-based point-of-care assessment. AIDS Behav. 2017 https://doi.org/10.1007/s10461-017-1718-5

44. Callon W, Saha S, Korthuis PT, et al. Which clinician questions elicit accurate disclosure of antiretroviral non-adherence when talking to patients? AIDS Behav. 2016;20(5):1108-15.

45. Wilson IB. Talking with your patients about medication adherence. Script Your Future, http://www.scriptyourfuture.org/medication-adherence-in-practice-webinar/. 2015. Accessed 21 Aug 2017.

46. Steiner JF. Using Adherence Information to Improve Care: From Clinic Visits to Populations. J Gen Intern Med. 2016;31(11):1272-3.

47. Lester RT, Ritvo P, Mills EJ, et al. Effects of a mobile phone short message service on antiretroviral treatment adherence in Kenya (WelTel Kenya1): a randomised trial. Lancet. 2010;376(9755):1838-45.

48. Choudhry NK, Bykov K, Shrank WH, Toscano M, Rawlins WS, Reisman L, et al. Eliminating medication copayments reduces disparities in cardiovascular care. Health Aff (Millwood). 2014;33(5):863-70.

49. Coca A, Agabiti-Rosei E, Cifkova R, Manolis AJ, Redon J, Mancia G. The polypill in cardiovascular prevention: evidence, limitations and perspective - position paper of the European Society of Hypertension. J Hypertens. 2017;35(8):1546-53.

50. Siegel SJ. Extended release drug delivery strategies in psychiatry: theory to practice. Psychiatry. 2005;2(6):22-31.

51. Krumme AA, Isaman DL, Stolpe SF, Dougherty S, Choudhry NK. Prevalence, effectiveness, and characteristics of pharmacy-based medication synchronization programs. Am J Manag Care. 2016;22(3):179-86.

52. Girdish C, Shrank W, Freytag S, et al. The impact of a retail prescription synchronization program on medication adherence. J Am Pharm Assoc (2003). 2017. 
53. Choudhry NK, Krumme AA, Ercole PM, et al. Effect of reminder devices on medication adherence: the REMIND randomized clinical trial. JAMA Intern Med. 2017;177(5):624-31.

54. Viswanathan $\mathbf{M}$, Golin $\mathbf{C E}$, Jones $\mathbf{C D}$, et al. Interventions to improve adherence to self-administered medications for chronic diseases in the United States: a systematic review. Annals of internal medicine. 2012;157(11):785-95.

55. Kronish IM, Moise N. In search of a "magic pill" for medication nonadherence. JAMA Intern Med. 2017;177(5):631-2.

56. DeKoekkoek T, Given B, Given CW, Ridenour $\mathbf{K}$, Schueller $\mathbf{M}$, Spoelstra SL. mHealth SMS text messaging interventions and to promote medication adherence: An integrative review. Journal of Clinical Nursing. 2015;24(19-20):2722-35.

57. Davidson TM, McGillicuddy J, Mueller M, et al. Evaluation of an mHealth medication regimen self-management program for African American and Hispanic uncontrolled hypertensives. J Pers Med. 2015;5(4):389-405.

58. Kronish IM, Moise N, McGinn T, et al. An electronic adherence measurement intervention to reduce clinical inertia in the treatment of uncontrolled hypertension: the MATCH cluster randomized clinical trial. J Gen Intern Med. 2016:31(11):1294-300.

59. Wilson IB, Laws MB, Safren SA, et al. Provider-focused intervention increases adherence-related dialogue but does not improve antiretroviral therapy adherence in persons with HIV. J Acquir Immune Defic Syndr. 2010a;53(3):338-47.

60. Jones DL, Sued O, Cecchini D, et al. Improving adherence to care among "hard to reach" HIV-infected patients in Argentina. AIDS Behav. 2016;20(5):987-97.

61. Wilson SR, Strub P, Buist AS, et al. Shared treatment decision making improves adherence and outcomes in poorly controlled asthma. Am J Respir Crit Care Med. 2010b; 181(6):566-77.

62. Adams AS, Uratsu C, Dyer $\mathbf{W}$, et al. Health system factors and antihypertensive adherence in a racially and ethnically diverse cohort of new users. JAMA Intern Med. 2013;173(1):54-61.

63. Horberg MA, Hurley LB, Towner WJ, et al. Determination of optimized multidisciplinary care team for maximal antiretroviral therapy adherence. J Acquir Immune Defic Syndr. 2012;60(2):183-90. 